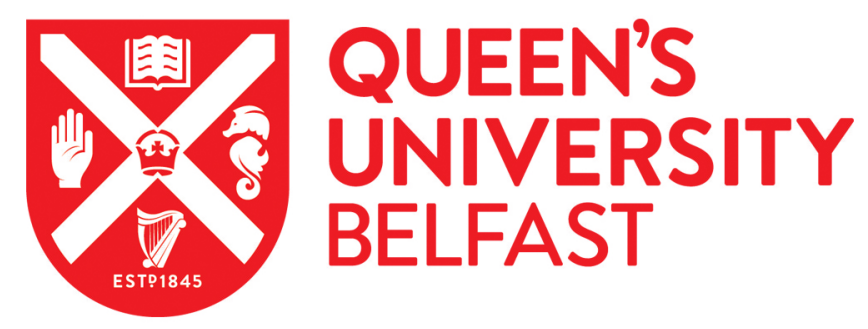

\title{
Power-law energy distributions of small-scale impulsive events on the active Sun: results from IRIS
}

\author{
Vilangot Nhalil, N., Nelson, C. J., Mathioudakis, M., Doyle, J. G., \& Ramsay, G. (2020). Power-law energy \\ distributions of small-scale impulsive events on the active Sun: results from IRIS. Monthly Notices of the Royal \\ Astronomical Society, 499, 1385-1394. https://doi.org/10.1093/mnras/staa2897
}

Published in:

Monthly Notices of the Royal Astronomical Society

Document Version:

Publisher's PDF, also known as Version of record

Queen's University Belfast - Research Portal:

Link to publication record in Queen's University Belfast Research Portal

\section{Publisher rights}

Copyright 2020 The Author(s). Published by Oxford University Press on behalf of the Royal Astronomical Society.

This work is made available online in accordance with the publisher's policies. Please refer to any applicable terms of use of the publisher.

\section{General rights}

Copyright for the publications made accessible via the Queen's University Belfast Research Portal is retained by the author(s) and / or other copyright owners and it is a condition of accessing these publications that users recognise and abide by the legal requirements associated with these rights.

Take down policy

The Research Portal is Queen's institutional repository that provides access to Queen's research output. Every effort has been made to ensure that content in the Research Portal does not infringe any person's rights, or applicable UK laws. If you discover content in the Research Portal that you believe breaches copyright or violates any law, please contact openaccess@qub.ac.uk. 


\title{
Power-law energy distributions of small-scale impulsive events on the active Sun: results from IRIS
}

\author{
Nived Vilangot Nhalil ${ }^{\oplus},{ }^{1,2 \star}$ Chris J. Nelson ${ }^{\oplus},{ }^{2}$ Mihalis Mathioudakis, ${ }^{2}$ J. Gerry Doyle ${ }^{1}$ \\ and Gavin Ramsay ${ }^{1}$ \\ ${ }^{1}$ Armagh Observatory \& Planetarium, College Hill, Armagh BT61 9DG, UK \\ ${ }^{2}$ Astrophysics Research Centre (ARC), School of Mathematics and Physics, Queens University, Belfast BT7 1NN, Northern Ireland, UK
}

Accepted 2020 September 12. Received 2020 September 12; in original form 2020 June 5

\begin{abstract}
Numerous studies have analysed inferred power-law distributions between frequency and energy of impulsive events in the outer solar atmosphere in an attempt to understand the predominant energy supply mechanism in the corona. Here, we apply a burst detection algorithm to high-resolution imaging data obtained by the Interface Region Imaging Spectrograph to further investigate the derived power-law index, $\gamma$, of bright impulsive events in the transition region. Applying the algorithm with a constant minimum event lifetime (of either $60 \mathrm{~s}$ or $110 \mathrm{~s}$ ) indicated that the target under investigation, such as Plage and Sunspot, has an influence on the observed power-law index. For regions dominated by sunspots, we always find $\gamma<2$; however, for data sets where the target is a plage region, we often find that $\gamma>2$ in the energy range $\left(\sim 10^{23}, \sim 10^{26}\right)$ erg. Applying the algorithm with a minimum event lifetime of three time-steps indicated that cadence was another important factor, with the highest cadence data sets returning $\gamma>2$ values. The estimated total radiative power obtained for the observed energy distributions is typically 10-25 percent of what would be required to sustain the corona indicating that impulsive events in this energy range are not sufficient to solve coronal heating. If we were to extend the power-law distribution down to an energy of $10^{21} \mathrm{erg}$, and assume parity between radiative energy release and the deposition of thermal energy, then such bursts could provide 25-50 per cent of the required energy to account for the coronal heating problem.
\end{abstract}

Key words: methods: observational - methods: statistical - techniques: spectroscopic-Sun: activity -Sun: atmosphere-Sun: corona.

\section{INTRODUCTION}

How the coronae of the Sun and solar-like stars are heated to multimillion-degree temperatures remains an open question in modern astrophysics. Typically, energy supply is explained as coming either from magnetohydrodynamic waves (Alfvén 1947) or from magnetic reconnection (Parker 1988). However, because the spatial scales of dissipation from either mechanism would be so small (km length-scales or less), neither has currently been shown to be dominant in the Sun and other solar-type stars. It is well known that large-scale solar flares are magnetic reconnection events, which can release huge amounts of energy $\left(\sim 10^{32} \mathrm{erg}\right)$ over time-scales of the order of minutes, yet these events are sufficiently rare that their time-averaged energy contribution is not high enough to compensate for the radiative losses of the corona. On sub-arcsecond scales, a host of magnetic reconnection-associated features that include, but are not limited to, Ellerman bombs (EBs; Vissers, Rouppe van der Voort \& Rutten 2013; Nelson et al. 2015; Reid et al. 2016; Ellerman 1917), Quiet-Sun Ellerman-like Brightenings (QSEBs; Rouppe van der Voort, Rutten \& Vissers 2016; Nelson et al. 2017), Explosive Events (EEs; Brueckner \& Bartoe 1983; Huang et al. 2017, 2018), and UV bursts (Peter et al. 2014; Nelson, Doyle \& Erdélyi 2016;

^E-mail: nived.vilangot.nhalil@armagh.ac.uk
Young et al. 2018) have been shown to be common in the solar atmosphere, but the ability of these features to deposit enough energy to heat the upper layers of the Sun remains uncertain.

Two numbers are often cited by researchers when discussing the ability of magnetic reconnection events to provide the $10^{7} \mathrm{erg} \mathrm{s}^{-1} \mathrm{~cm}^{-2}$ required to heat the upper layers of the active solar atmosphere (Withbroe \& Noyes 1977). The first is that in order to understand whether magnetic reconnection is relevant to coronal heating, we must be able to probe 'nano-flare' events with energies less than $\sim 10^{24} \mathrm{erg}$ (Parker 1988). This work built on Levine (1974), who proposed that coronal heating was due to a multitude of small reconnections. Despite the apparent simplicity of this task, it has so far proved difficult to identify large samples of events at these energies due to the observational stipulations required. Obtaining both the high spatial and temporal resolution needed in the upper layers of the solar atmosphere with a large enough field of view (FOV) to provide some general information is still a non-trivial task. The second number often quoted is that in order to explain the heating of the corona by magnetic reconnection events, the energy versus frequency distribution must have a power law with an index, $\gamma$, steeper than 2 (Hudson 1991). If $\gamma>2$, magnetic reconnection events with lower energies are important in heating the corona; however, if $\gamma<2$, higher energy flares are dominant and lower energy events such as nano-flares cannot contribute much to the total power (Krucker \& Benz 1998; Parnell \& Jupp 2000). 
Therefore, investigating the frequencies of suitably small impulsive burst events (often assumed to be an observational signature of magnetic reconnection) in the upper atmosphere remains an essential task in solar physics.

Numerous authors have attempted to analyse the power-law distributions of brightenings in the upper solar atmosphere using a variety of data. Shimizu (1995), for example, looked at active region (AR) transient brightenings and found that their frequency distribution as a function of energy had a power law with an index of 1.64-1.89. Interestingly, it was found that the returned power-law index was dependent upon the pixel size, with smaller pixels providing larger indices. Notably though, the events studied by Shimizu (1995) had energies in excess of $10^{27}$ erg, well above the $10^{24}$ erg level suggested by Parker (1988). Parnell \& Jupp (2000) investigated Transition Region and Coronal Explorer (TRACE; Golub et al. 1999) events with energies in the range of $10^{23}-10^{26}$ erg deriving a power-law index greater than 2. However, those authors suggested that the input from this energy range was insufficient to heat the quiet solar corona, implying that even smaller energies, down to $10^{21} \mathrm{erg}$, would be required. Aschwanden \& Parnell (2002) expanded on this work and looked at TRACE data from the $171 \AA$ and $195 \AA$ filters deriving power-law indices of 1.86 and 1.81, respectively. The above dependence on instrument and pixel scale highlights the importance of temporal and spatial resolution in investigating the smallest and faintest events.

In almost all observational studies conducted to date, the derived power-law index between frequency and energy has not been sufficient to provide enough energy to the upper atmosphere to compensate for radiative losses. There is, therefore, currently very little confirmatory evidence to support the hypothesis that micro/nanoflaring is sufficient to heat the coronae. Analytical and numerical modelling by a host of authors (e.g. Cargill \& Klimchuk 2004; Rappazzo et al. 2008; Parnell, Maclean \& Haynes 2010; Bowness, Hood \& Parnell 2013; Jess et al. 2019; Mondal, Oberoi \& Mohan 2020) offers some hints that further observational studies would be beneficial. Additionally, research on solar-like stars could offer some clues about the contribution of magnetic reconnection to the heating of the upper atmosphere. For example, Doyle \& Butler (1985) presented a linear correlation between the X-ray flux of quiescent dMe stars (plus the Sun) against the time-averaged energy emitted by flares in a near ultra-violet waveband. Various authors have looked at AR mostly when studying nano-flaring (e.g. Antiochos et al. 2003; Testa et al. 2013; Graham, De Pontieu \& Testa 2019) finding constraints on parameters, such as the time interval between heating events in a flux tube and the variation in magnitude between events.

Here, we look again at this problem using high spatial and temporal resolution observations obtained by the Interface Region Imaging Spectrograph (IRIS; De Pontieu et al. 2014). This instrument has an effective spatial resolution of $0.33^{\prime \prime}$, allowing us to probe the transition region of the Sun down to sub-arcsecond scales. Our work is set out as follows: In Section 2, we outline the data studied here and the detection algorithm employed. In Section 3, we present the results obtained through applying our algorithm, and in Section 4, we include a brief discussion and draw our conclusions.

\section{METHODS}

\subsection{Observations}

The aim of this work is to analyse burst events with short lifetimes in the transition region of ARs meaning high cadence $(\lesssim 20 \mathrm{~s})$ in the Slit-Jaw Imager (SJI) $1400 \AA$ channel is essential. Additionally, we require a near constant FOV in order to analyse the entire lifetimes of bursts implying that data must have been recorded either in sit-andstare mode (in which the IRIS slit is fixed at a particular location on the Sun and continuously tracks the solar rotation) or in dense raster mode with a maximum of four steps $\left(\sim 1^{\prime \prime}\right.$ shift in FOV). Searching the IRIS data catalogue with these criteria returned several hundred candidate data sets totalling tens of Terabyte (TB) in size. However, as analysing all of these data sets was not possible at this time, here we focus instead on a smaller sample of 12 data sets in order to understand some general properties of bursts, which can be used to infer interesting results and direct future research. The details of these 12 SJI data sets are presented in Table 1, where the stars indicate the five data sets, which also include simultaneous imaging from the IRIS SJI $1330 \AA$ channel. These data were downloaded as Level-2 IRIS data products on which dark current removal, flat-field, geometrical distortion, and orbital and thermal drift corrections had been applied.

The SJI $1400 \AA$ A channel is dominated by the Si IV $1394 \AA$ A and 1403 $\AA$ resonance lines, which are formed in the transition region, while the SJI $1330 \AA$ A channel is dominated by the C II $1335 \AA$ and $1336 \AA$ lines, which are formed in the upper chromosphere. The mean formation temperatures of $\mathrm{C}$ II and Si IV lines are $\sim 3 \times 10^{4} \mathrm{~K}$ and $\sim 8 \times 10^{4}$ K, respectively (Rathore \& Carlsson 2015; Rathore et al. 2015). Therefore, these two SJI channels provide an opportunity to study the energetics of burst-like events in two different layers in the solar atmosphere. Numerous authors, e.g. Ayres et al. (1995), have shown flux-flux relationships between transition region lines and the X-ray flux. This work was taken one step further by Bruner \& McWhirter (1988) and Doyle (1996), who showed a linear relationship between the flux of transition region lines and coronal lines, and the total radiative losses; hence, the Si IV $1394 \AA$ line is a good proxy to estimate the radiative losses in the atmosphere.

Seven out of the 12 data sets were already binned with a pixel scale of $0.33^{\prime \prime}$, while the pixel scale for the remaining observations was approximately $0.17^{\prime \prime}$. In order to have consistent pixel scales in all data sets, all observations were binned to have a pixel scale of $0.33^{\prime \prime}$ before the application of the detection algorithm. In Fig. 1, we show an example of a data set selected for analysis here. This data set (Set 1 from Table 1) sampled a sunspot within AR NOAA 11836 in sit-and-stare mode between 15:59:35 UT and 17:54:29 UT on 2013 August 31. Both $1330 \AA$ and $1400 \AA$ SJ images were recorded with a 12 -s cadence and a pixel size of $0.17^{\prime \prime}$. The exposure time for the SJI observations was 4 s. In panels (a) and (b), we plot the SJI $1400 \AA$ and $1330 \AA$ images of the AR sampled at 17:12:28 UT, respectively. Corresponding AIA $1600 \AA$ and $193 \AA$ images collected by the Solar Dynamics Observatory's Atmospheric Imaging Assembly (SDO/AIA Lemen et al. 2012) instrument are shown in panel (c) and panel (d), respectively. The white box overlaid on panels (c) and (d) outlines the FOV of the IRIS SJI observation. The SJI data in Fig. 1 clearly display the presence of the localized brightenings we aim to study here.

\subsection{Burst detection algorithm}

\subsubsection{Burst selection}

Intensities in IRIS SJI data are stored in DN units; however, the exposure time of images may not be constant during the observation. In order to correct for this effect, intensity values were divided by the corresponding exposure times before these exposure normalized images were used for the detection of impulsive brightening events. Event selection was carried out by scanning the light curve of each pixel in the FOV. The algorithm starts by calculating the background intensity in each pixel from its time series data before 
Table 1. Details of the IRIS SJI observations studied here. The 'Cadence (s)' column refers to the IRIS SJI $1400 \AA$ A channel cadence. The $*$ indicates that IRIS 1330 A SJI data are also available with the same cadence.

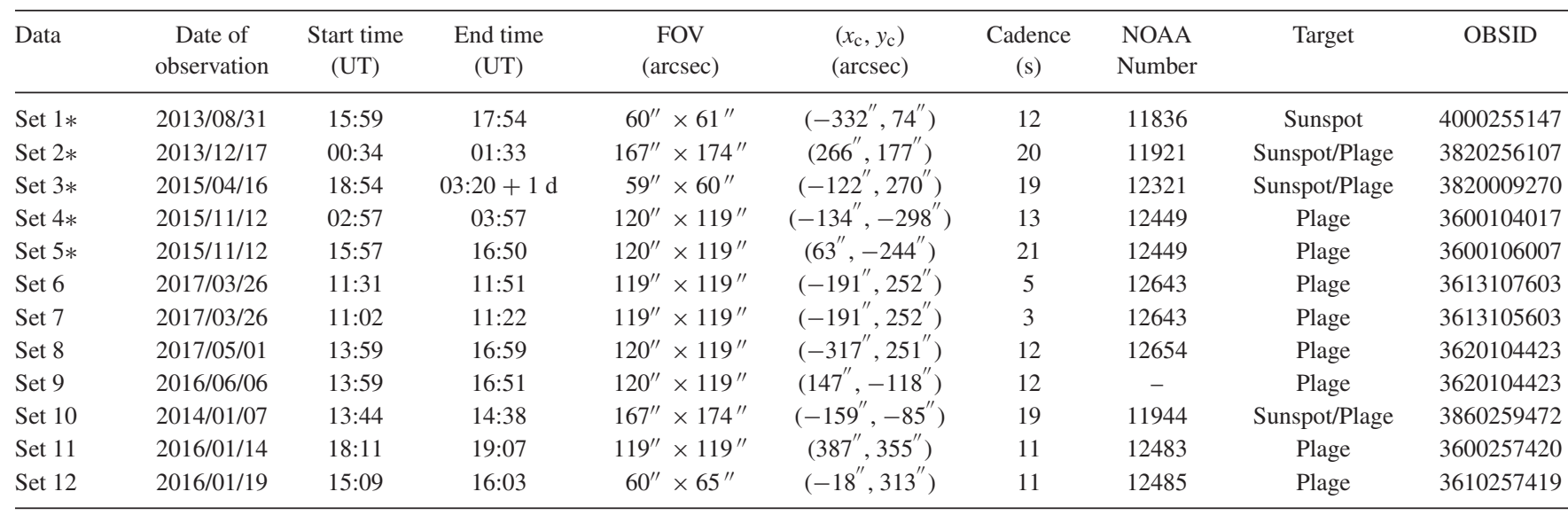

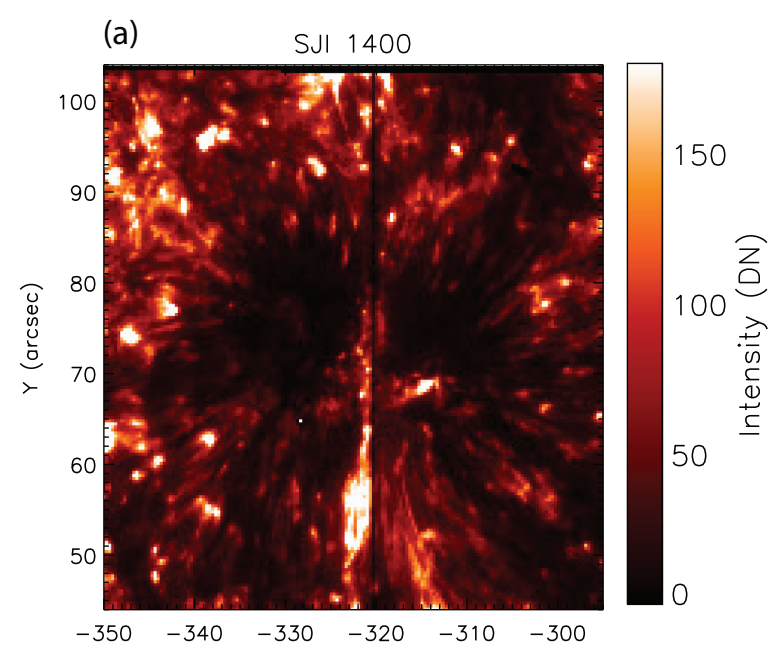

(b)

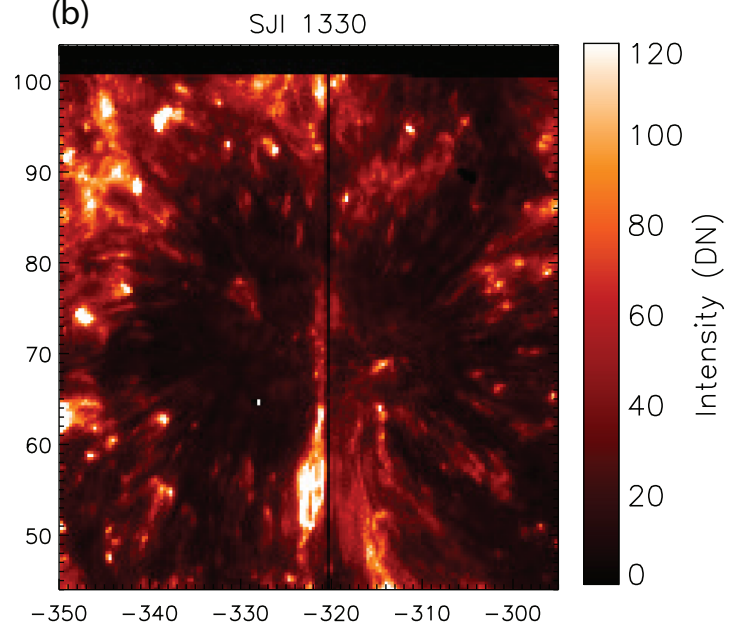

(c)

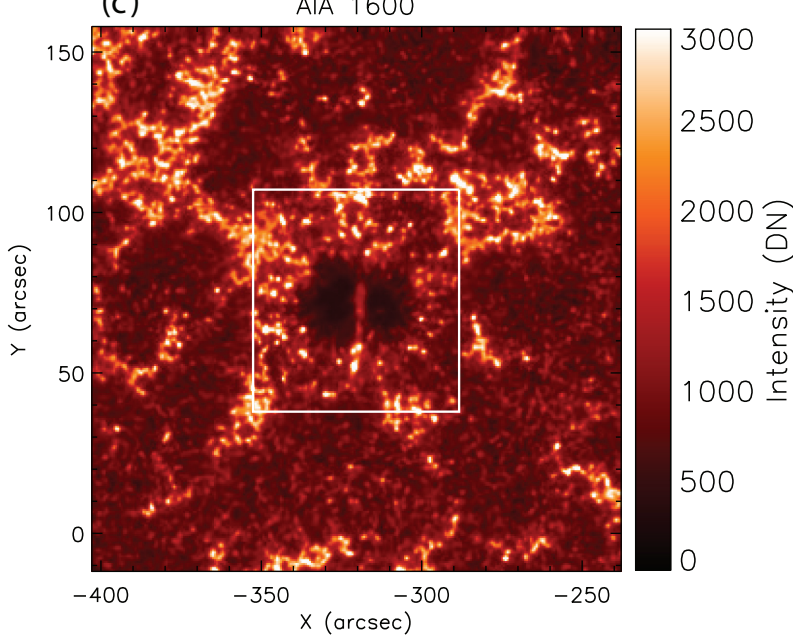

(d)

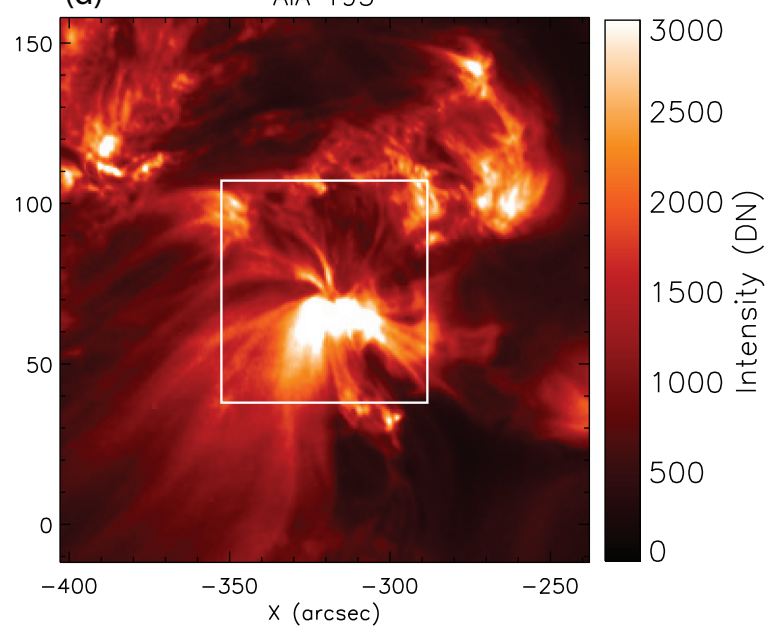

Figure 1. The studied FOV within AR 11836 (data set 1) sampled on August 312013 at approximately 15:59 UT. The SJI $1400 \AA$ and $1330 \AA$ images are plotted in panels (a) and (b), respectively. Context imaging from the SDO/AIA AIA $1600 \AA$ (c) and $193 \AA$ (d) filters is also plotted. The white box overlaid on these panels identifies the FOV of the IRIS SJI observation.

obtaining the standard deviation $(\sigma)$ with respect to the background intensity. The algorithm first determines the minimum intensity of the pixel from its light curve and then calculates the average intensity of the light curve by excluding regions which are five times larger than the minimum intensity. This condition helps to remove impulsive brightening while calculating the background intensity. Impulsive bursts were then identified through the presence of intensity peaks that are significantly above background fluctuations for a 


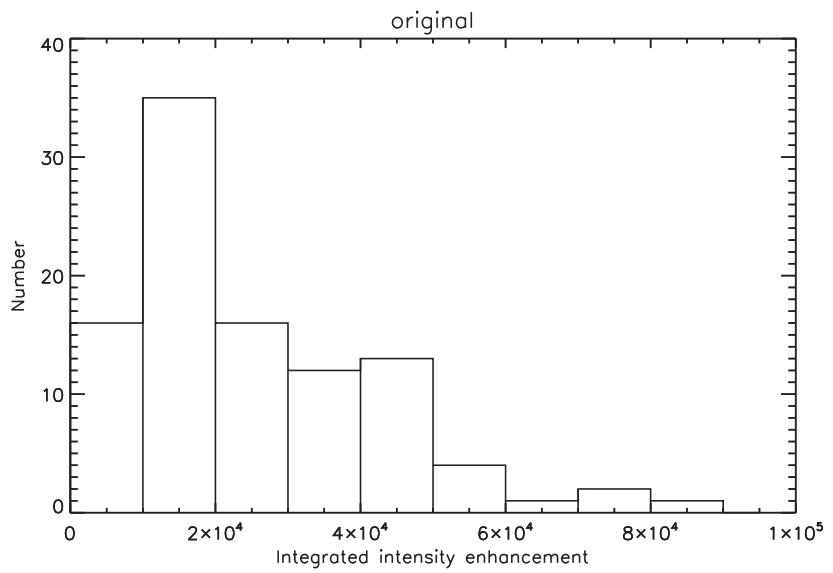

detected

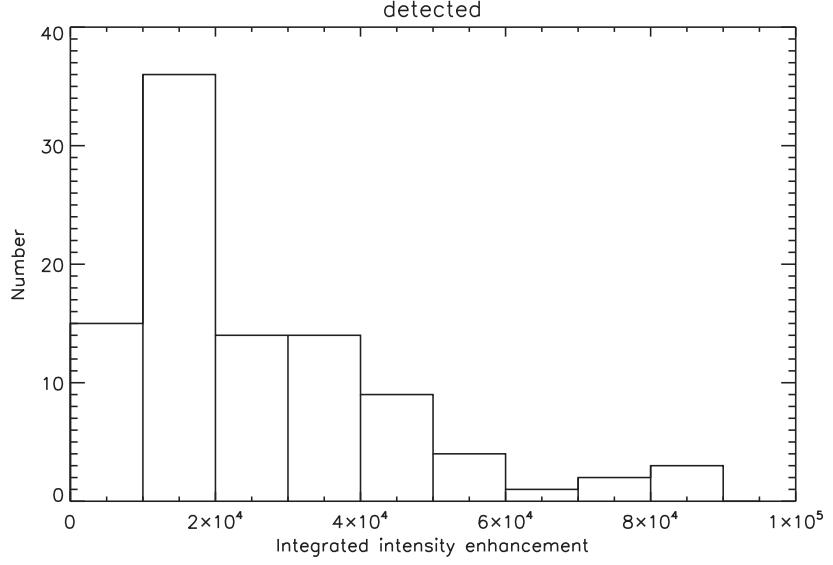

Figure 2. Top panel shows the histogram for the actual value of integrated intensity enhancement in the burst. A similar plot is shown in the bottom panel but for bursts detected using $3 \sigma$ detection condition.

user-defined time. We analysed the effects of varying the local intensity threshold between $3 \sigma$ and $7 \sigma$ as well as varying the minimum lifetime of bursts with these results being presented in the later sections of this article. The minimum burst lifetime studied was limited to three times the cadence in order to differentiate between bursts and cosmic ray spikes, which typically do not appear at the same location in subsequent images.

We also applied a condition that the enhanced emission identified as bursts should be at least twice the average intensity of the entire FOV. A single pixel can have multiple bursts since there is a possibility of having multiple-intensity maxima at the same location through time. As there could be some non-impulsive brightening events in the FOV, an impulsivity criterion was applied to remove sustained brightening events. For this, we calculated the average enhancement in intensity during the burst and divided this value by the background flux for that specific pixel. This ratio should be greater than 1 to be considered as an impulsive event. As no algorithm is perfect in detecting bursts, there is a possibility of excluding actual burst-like events as well as including non-burst regions. Choosing the correct combinations of parameters and conditions can minimize the mis-identification of the bursts.

\subsubsection{Area and lifetime of the burst}

Once an impulsive burst was identified in a pixel, an iterative approach was then used to calculate the total area and lifetime of the event. In the first step, the algorithm searched for connected pixels with intensity above the user-defined intensity threshold during the peak time. Minimum area thresholding was applied within the algorithm to reduce incorrect identifications, with limits of $>1,>2$, or $>3$ pixels all studied. In the next step, the algorithm searched for the impulsive events connected to the pixel in the previous time-step. The algorithm continued this iteration until it reached a time where there were no pixels above the intensity threshold. This same iterative method was also applied forward in time. Using this method, we were able to determine the starting time as well as the ending time of the burst. In this approach, the total area of the burst is a combination of all the spatially connected pixels throughout its lifetime. Once a burst location and time were identified, we removed these data points from the data cube to avoid multiple detections.

\subsubsection{Energy determination}

Before calculating the energy of these impulsive events, it is necessary to express the SJI intensities in radiometric units, which provides the energy flux at the Sun. This can be done using the equation:

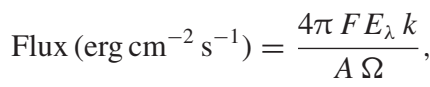

where $F$ is the flux in DN/s, $E_{\lambda}$ is the energy of the photon, and $k$ is the factor that converts the DN to the number of photons. This factor is the ratio between gain and yield. The gain is the number of electrons released in the detector that yield $1 \mathrm{DN}$ and the yield is the number of electrons released by one incident photon. $\Omega$ is the SJI pixel size in steradian. $A$ is the post-launch effective area calculated using the 'iris_get_response.pro' routine, which accounts for the degradation of the instrument since launch. The time over which the energy was calculated included the rise and decay phases of the bursts, delineated by the times when the gradient of the pixel intensity was zero before and after the burst. The energy radiated during the event can be calculated by integrating the radiated energy flux in time in all bright pixels of each event. The background emission was subtracted while calculating the energy of the burst.

\subsection{Testing the detection algorithm}

Before performing the detection on real SJ images, we tested the detection algorithm on a synthetic data set to verify its robustness. First, we created a data cube that has base intensities that follow a Gaussian distribution. Since real solar data are not perfectly Gaussian, we introduced asymmetry by multiplying each pixel by an error function. Secondly, we scanned through the light curve of each pixel to obtain the average background intensity and the background fluctuations. Thirdly, we randomly selected 100 locations in the data cube using the IDL randomu function and increased the intensity values in the corresponding locations by $10 \sigma$ for a specified lifetime. These enhanced intensity pixels represent the bursts. Finally, we detected bursts with a minimum area of 3 pixels that are $3 \sigma$ above the background and obtained the integrated intensity enhancement.

The algorithm was successful in detecting simulated bursts, detecting 98 bursts out of 100 . The code failed to detect two bursts since they were overlapping. In Fig. 2, we compare the intensity enhancement integrated over the actual area and lifetime of the bursts with the one obtained from the detected bursts. The integrated intensity enhancement represents the energy of the burst, which determines the power-law slope. Histograms for the actual values of integrated intensity enhancement are shown in the top panel of Fig. 2, while the value determined from the detected bursts is presented in the bottom panel. The similarity between these two plots implies how successful is the detection algorithm in determining the energies of the bursts. 

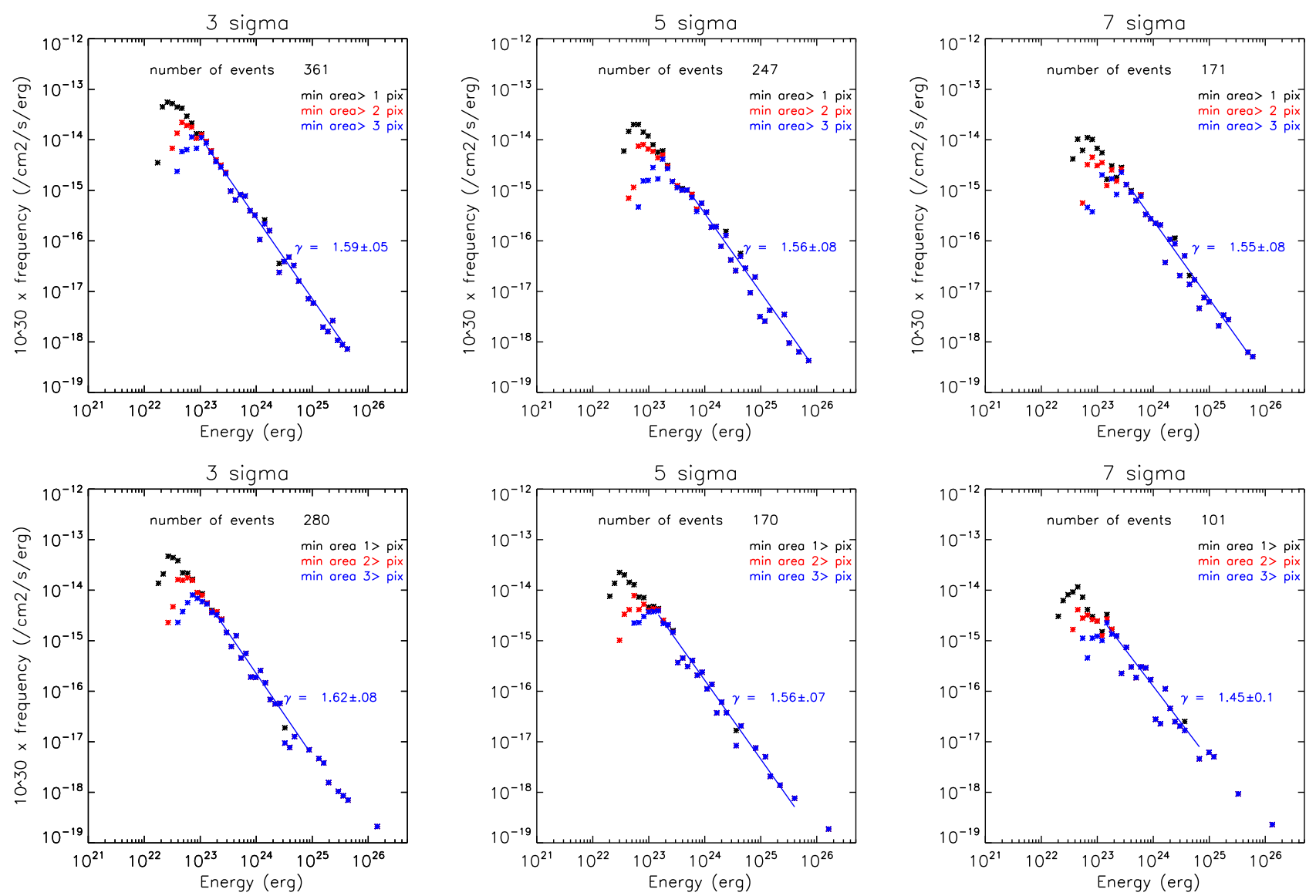

Figure 3. The effect of changing the intensity threshold from $3 \sigma$ (left column) to $5 \sigma$ (middle column) to $7 \sigma$ (right column) above the background, for varying minimum areas, on the detection of impulsive bursts in Data set 1 for the SJI $1400 \AA$ (top row) and $1330 \AA$ (bottom row) channels. The distribution of bursts with area $>1,>2$, and $>3$ pixels is shown in black, red, and blue, respectively. The effects of the minimum area condition are most apparent at low energies (below approximately $10^{23} \mathrm{erg}$ ). The overlaid 'number of events' indicates the total number of events detected with the area $>3$ pix.

Table 2. Power-law indices of the impulsive events detected in each data set by defining bursts as peaks with intensity enhancement $5 \sigma$ and $3 \sigma$ above the background for $\sim 110 \mathrm{~s}$ (second to fifth columns) and $\sim 60 \mathrm{~s}$ (sixth to ninth columns). 'N/A' indicates that too few events were returned to provide an accurate estimate of the power-law index.

\begin{tabular}{|c|c|c|c|c|c|c|c|c|}
\hline \multirow[t]{3}{*}{ Data } & $\begin{array}{c}\text { Power law }(\gamma) \\
\text { SJI } 1400\end{array}$ & $\begin{array}{c}\text { Power law }(\gamma) \\
\text { SJI } 1330\end{array}$ & $\begin{array}{c}\text { Power law }(\gamma) \\
\text { SJI } 1400\end{array}$ & $\begin{array}{c}\text { Power law }(\gamma) \\
\text { SJI } 1330\end{array}$ & $\begin{array}{c}\text { Power law }(\gamma) \\
\text { SJI } 1400\end{array}$ & $\begin{array}{c}\text { Power law }(\gamma) \\
\text { SJI } 1330\end{array}$ & $\begin{array}{c}\text { Power law }(\gamma) \\
\text { SJI } 1400\end{array}$ & $\begin{array}{c}\text { Power law }(\gamma) \\
\text { SJI } 1330\end{array}$ \\
\hline & \multicolumn{4}{|c|}{$110 \mathrm{~s}$} & \multicolumn{4}{|c|}{$60 \mathrm{~s}$} \\
\hline & \multicolumn{2}{|c|}{$5 \sigma$ above the background } & \multicolumn{2}{|c|}{$3 \sigma$ above the background } & \multicolumn{2}{|c|}{$5 \sigma$ above the background } & \multicolumn{2}{|c|}{$3 \sigma$ above the background } \\
\hline Set 1 & $1.56 \pm 0.08$ & $1.56 \pm 0.09$ & $1.59 \pm 0.05$ & $1.62 \pm 0.08$ & $1.54 \pm 0.05$ & $1.68 \pm 0.08$ & $1.81 \pm 0.04$ & $1.66 \pm 0.04$ \\
\hline Set 2 & $1.62 \pm 0.04$ & $1.36 \pm 0.04$ & $1.72 \pm 0.03$ & $1.57 \pm 0.04$ & $1.74 \pm 0.02$ & $1.59 \pm 0.04$ & $1.96 \pm 0.02$ & $1.81 \pm 0.02$ \\
\hline Set 3 & $1.71 \pm 0.04$ & $1.55 \pm 0.06$ & $1.89 \pm 0.03$ & $1.74 \pm 0.05$ & $2.01 \pm 0.03$ & $1.71 \pm 0.04$ & $2.03 \pm 0.02$ & $1.99 \pm 0.03$ \\
\hline Set 6 & N/A & - & N/A & - & N/A & - & $1.62 \pm 0.20$ & - \\
\hline Set 7 & N/A & - & N/A & - & $1.62 \pm 0.20$ & - & $1.75 \pm 0.31$ & - \\
\hline Set 8 & $1.83 \pm 0.02$ & - & $1.83 \pm 0.03$ & - & $1.90 \pm 0.02$ & - & $1.91 \pm 0.02$ & - \\
\hline Set 9 & $1.93 \pm 0.03$ & - & $1.82 \pm 0.02$ & - & $1.94 \pm 0.03$ & - & $1.94 \pm 0.02$ & - \\
\hline Set 10 & $1.63 \pm 0.07$ & - & $1.76 \pm 0.04$ & - & $1.78 \pm 0.04$ & - & $1.98 \pm 0.02$ & - \\
\hline Set 11 & $1.89 \pm 0.16$ & - & $2.06 \pm 0.12$ & - & $1.97 \pm 0.07$ & - & $2.03 \pm 0.05$ & - \\
\hline
\end{tabular}

\section{RESULTS}

Initially, we tested the effects of the chosen intensity threshold and minimum area conditions on the returned power-law indices. We present results from Data set 1 in Fig. 3; however, results from all other data sets are included in Table 2. Some data sets do not have the SJI $1330 \AA$ channel; also, for the short duration data sets, we have too few points to provide accurate estimates of the power-law index when using a minimum lifetime condition of $110 \mathrm{~s}$. In the top row of Fig. 3, we plot radiative energy against frequency for burst events detected in the SJI $1400 \AA$ channel with a minimum 

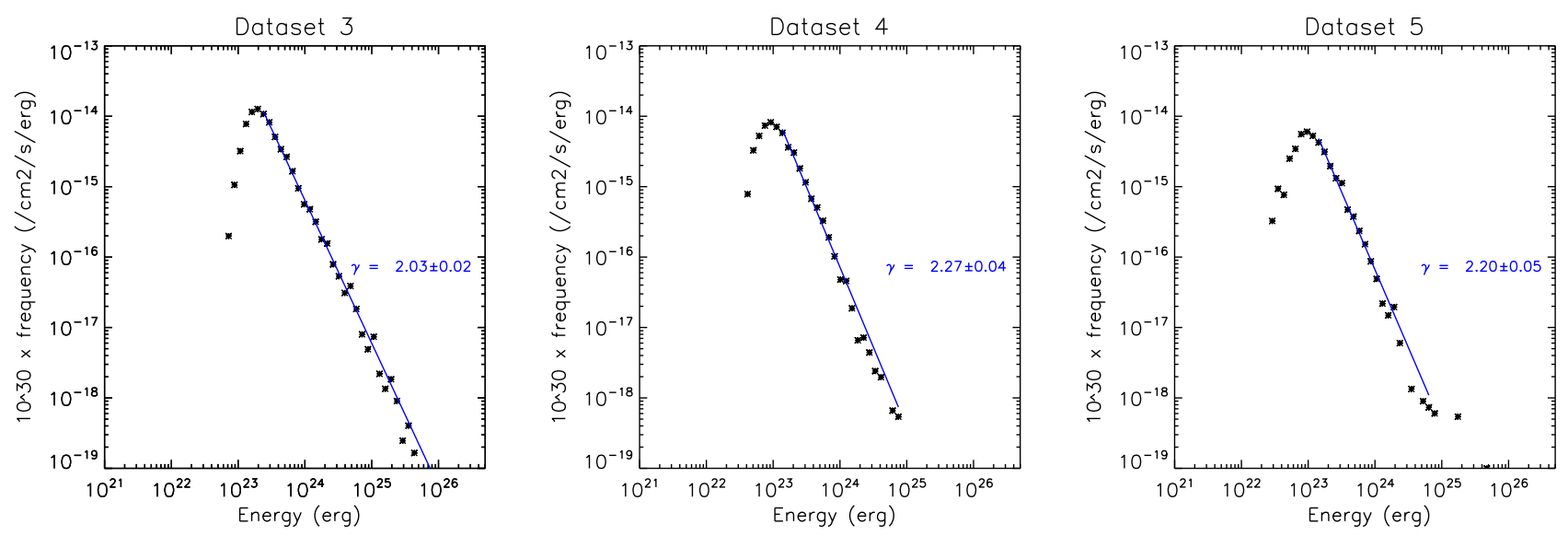

Figure 4. Power-law distributions of impulsive events detected in the SJI 1400 channel with intensity enhancements above $3 \sigma$, minimum areas of $>3$ pixels, and minimum lifetimes of $60 \mathrm{~s}$ for Data sets 3,4 , and 5 .

lifetime of $110 \mathrm{~s}$, with the three panels representing varying intensity thresholds. Similar results are plotted for the IRIS SJI $1330 \AA$ A channel in the bottom panels. The intensity threshold used in the algorithm is shown on the top of each panel, with the distribution of bursts detected with enhancements of $3 \sigma, 5 \sigma$, and $7 \sigma$ above the background being shown in the left, middle, and right columns, respectively. The colour of the distributions represents the minimum area condition applied while detecting impulsive events with areas of $>1,>2$, and $>3$ pixels being plotted in black, red, and blue, respectively. Intuitively, more events are detected (especially at lower energies) when lower intensity thresholds are applied. Notably, despite fewer events being recorded, the maximum energies returned from the SJI $1330 \AA$ channel are larger than those returned from the SJI $1400 \AA$ channel by up to an order of magnitude with some features having energies $>10^{26} \mathrm{erg}$ (see, for example, the middle panels of Fig. 3).

Here, the radiative energies of the detected impulsive events range from $10^{22} \mathrm{erg}$ to $10^{26} \mathrm{erg}$; thus, these events could be considered to be nano-flares. At lower energies $\left(<10^{23} \mathrm{erg}\right)$, the distribution of bursts fails to follow a power law as the frequency of the detected bursts decreases with decreasing energy. The drop-off from the power-law distribution at low energies is larger in bursts detected with less stringent intensity thresholds (black curves). This indicates that the drop-off is likely to be caused by observational limitations (e.g. spatial and temporal resolutions) as it becomes increasingly difficult to accurately determine the parameters of bursts that are near the detection limit of the instrument. It should be noted, however, that changes made in the distributions with varying minimum area conditions can be seen only in the lower energies with the blue curve being completely superimposed on the red and black curves at higher energies $\left(>10^{23} \mathrm{erg}\right)$.

As the transition region of ARs is generally very dynamic in nature, we note that if we reduce the intensity threshold too far, it is likely that fluctuations not associated with impulsive features will be counted as bursts. The above effect must be considered while calculating $\gamma$ for the distribution from each data set, meaning the power-law index was calculated only for energies above where the tail-off in frequencies is detected and for the most stringent area thresholding conditions (i.e. impulsive events with minimum area $>3$ pixels). For each of the intensity thresholds, the distribution displayed a clear linear behaviour on a logarithmic scale, with the resulting best fit and power-law index overlaid on each plot. Although the total number of events detected is reduced, as expected, with increasing sigma values, the $\gamma$ value remained stable within errors varying from $1.59 \pm 0.05$ $(3 \sigma)$ to $1.56 \pm 0.08(7 \sigma)$ for the IRIS SJI $1400 \AA$ channel and from $1.62 \pm 0.08(3 \sigma)$ to $1.45 \pm 0.11(7 \sigma)$ for the $1330 \AA$ channel. The error in slope represents the 1 sigma error associated with the model parameter. This consistency gives us confidence in our results at higher energies.

In Table 2, we display how the power-law index varies when impulsive events are identified with intensity enhancements of $5 \sigma$ and $3 \sigma$ above the background and lifetimes of over either $110 \mathrm{~s}$ (second to fifth columns) or $60 \mathrm{~s}$ (sixth to ninth columns) for both the SJI $1400 \AA$ and $1330 \AA$ channels. For the $1400 \AA$ channel with a minimum lifetime of $60 \mathrm{~s}, 4$ (2) out of 12 (10) data sets have powerlaw indices above 2 for $3 \sigma(5 \sigma)$. With a minimum lifetime of $110 \mathrm{~s}$, this decreases to $3(0)$ out of 10 (9) data sets. This result suggests that higher cadence data may return greater values of $\gamma$ in general. For the $1330 \AA$ channel (where only five data sets are available), these values are $1(0)$ out of 5 for a minimum lifetime of $110 \mathrm{~s}$ and 2 (1) out of 5 for a minimum lifetime of $60 \mathrm{~s}$. To display the accuracy of the power-law fitting, we plot three further examples (Data sets 3, 4 , and 5) from the SJI $1400 \AA$ line at the $3 \sigma$ level with a minimum lifetime of $60 \mathrm{~s}$ and a minimum area of 3 pixels in Fig. 4.

Our results also indicate that the type of solar structures within the selected region (e.g. sunspot or plage) influences the power-law index. For regions dominated by sunspots, we always find that $\gamma$ $<2$ for all threshold values when minimum lifetimes of $110 \mathrm{~s}$ and $60 \mathrm{~s}$ are considered; however, when the FOV is dominated by plage regions, the $\gamma$ values are higher, with three out of eight completely plage data sets (see Tables 1 and 2) having $\gamma>2$ in the SJI 1400 $\AA$ channel at the $3 \sigma$ level for both 110 -s and 60 -s cadences. We present results obtained from one plage region in more detail in Fig. 5. In the top row of Fig. 5, we plot an overview of the FOV as sampled by the SJI $1400 \AA$ channel (left-hand panel), the AIA 1600 $\AA$ channel (middle panel), and the AIA $193 \AA$ channel (right-hand panel). Large amounts of plage are evident but no sunspots or pores are present. Having examined the effects of the intensity threshold, minimum area, and solar structures within the FOV, we now move on to further investigate how the minimum lifetime criterion influences the returned $\gamma$ values.

Two data sets that sampled the plage region plotted in the top row of Fig. 5 were studied here, with both data sets being sampled at different cadences (Data sets 6 with $5 \mathrm{~s}$ and Data set 7 with $3 \mathrm{~s}$ ). The analysis of multiple data sets sampling the same FOV with different cadences allows us to remove the influence of different regions from 


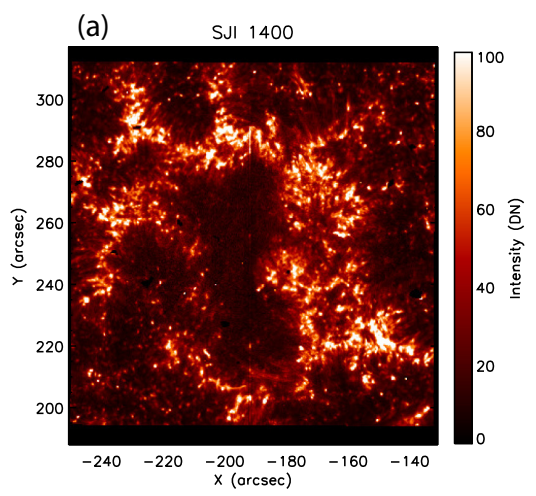

(b)
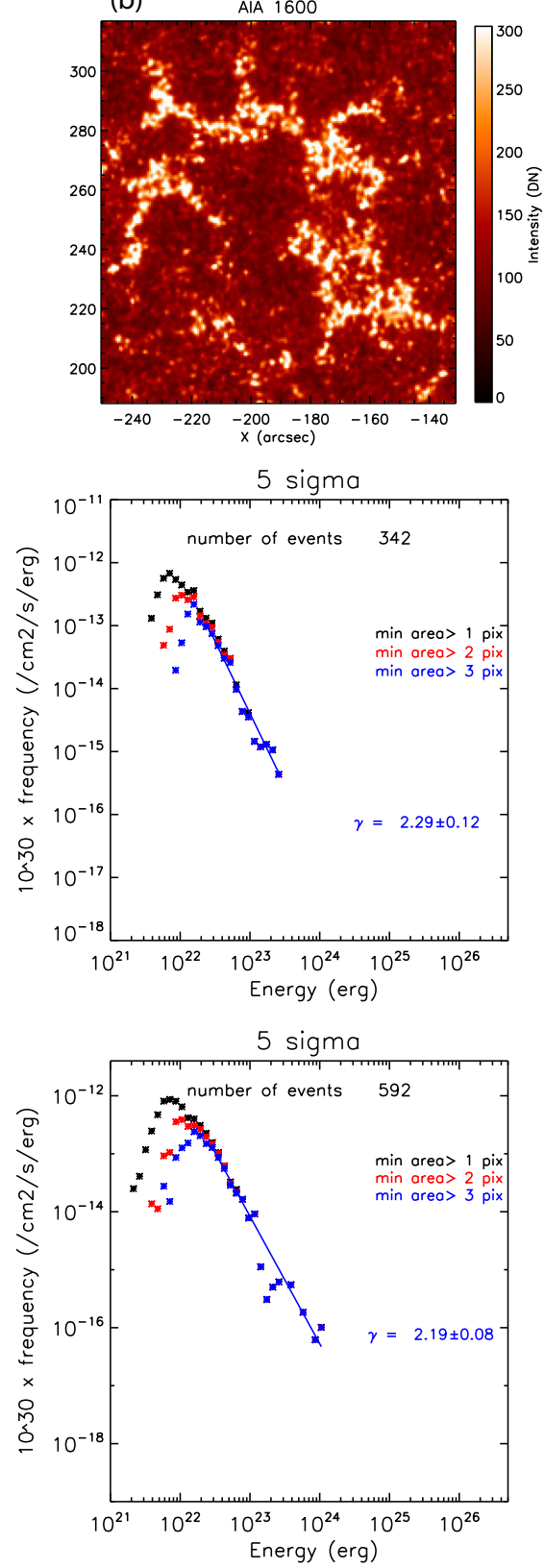
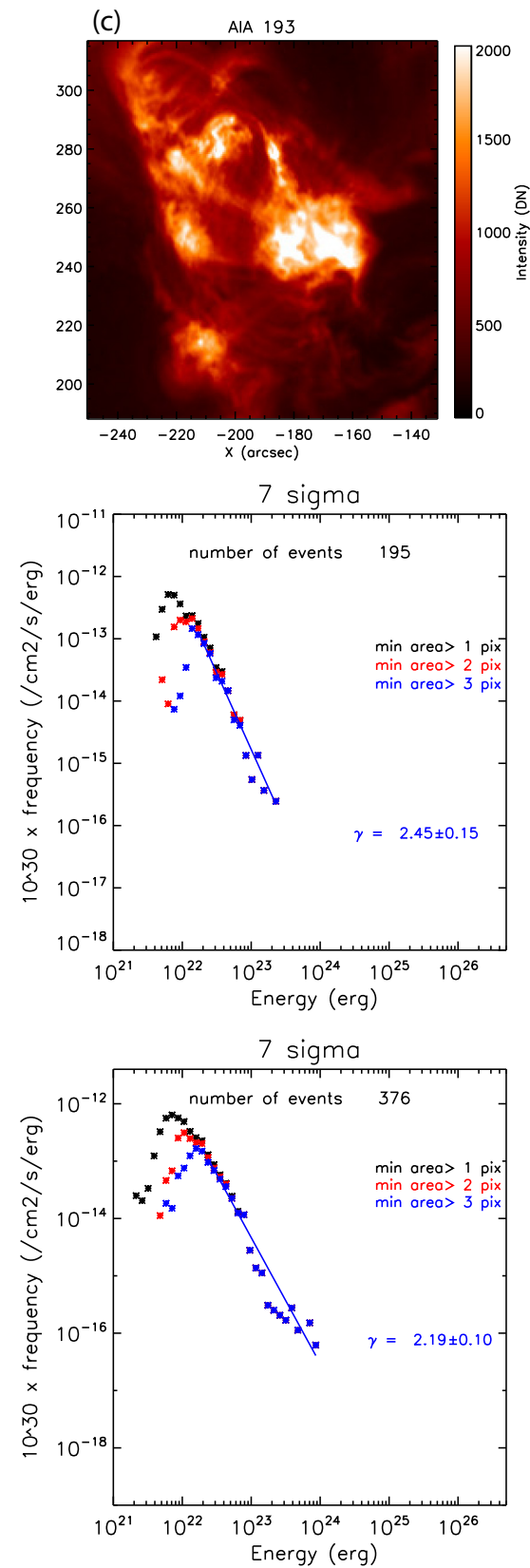

Figure 5. Results of applying the detection algorithm for a plage region (Data sets 6 and 7) for varying minimum lifetime thresholds. The top row plots SJI $1400 \AA$ (left-hand panel), AIA $1600 \AA$ (middle panel), and AIA $193 \AA$ (right-hand panel) context images displaying the clear plage structuring in this FOV. The middle row plots energy distributions of impulsive events detected using a minimum lifetime condition of $\sim 15 \mathrm{~s}$ (three time-steps) from Data set 6 . The left-hand panel, middle panel, and right-hand panel display the distributions of impulsive events detected using $3 \sigma, 5 \sigma$, and $7 \sigma$ detection limits, respectively. The colours of the distribution depict the minimum area condition as in Fig. 3. The bottom row plots the energy distributions of the impulsive events detected using a minimum lifetime condition of $\sim 9 \mathrm{~s}$ from Data set 7 . Again, the left-hand panel, middle panel, and right-hand panel display the impulsive events detected using $3 \sigma, 5 \sigma$, and $7 \sigma$ detection limits, respectively.

our analysis. These data sets did not contain SJI $1330 \AA$ data. In the middle and bottom rows of Fig. 5, we plot the power-law distributions of impulsive events returned for SJI $1400 \AA$ data from Data sets 6 (middle row) and 7 (bottom row) where the minimum lifetime condition is set to three times the cadence. For all sigma values, $\gamma>$ 2 for both data sets. Notably, the power-law indices obtained for these data sets with minimum lifetime conditions are significantly higher (above errors) than those calculated from the same data sets when a 110 -s or 60 -s minimum lifetime was applied. The power-law indices calculated for three times the cadence with intensity thresholding set at $3 \sigma$ are included in the fifth row of Table 3 for the remaining data sets. All but three data sets return $\gamma>2$.

We now move on to estimating the total radiative energies from each data set. The total power per unit area of each impulsive event from the SJI $1400 \AA$ emission, $P_{1400}$, can be calculated by integrating the events energy times the frequency of occurrence of events per unit area per unit time, $f(E)$. Since the frequency of occurrence of these impulsive events follows a power-law distribution, the power 
Table 3. Total radiative power obtained for all of the data sets using an intensity threshold of $3 \sigma$ and a minimum lifetime threshold of three times the cadence. $P_{\text {tot }}$ in the seventh column represents the total radiative power in the observed minimum and maximum energy range (see equation 2). In the eighth column, $P_{\text {tot }}$ is calculated for the energy range of $10^{21} \mathrm{erg}$ to observed maximum energy.

\begin{tabular}{lccccccc}
\hline Data & $\begin{array}{c}\text { Date of } \\
\text { observation }\end{array}$ & $\begin{array}{c}E_{\text {Min }} \\
(\mathrm{erg})\end{array}$ & $\begin{array}{c}E_{\text {Max }} \\
(\mathrm{erg})\end{array}$ & $\begin{array}{c}\text { Power-law } \\
\text { index }(\gamma)\end{array}$ & $\begin{array}{c}\text { Minimum } \\
\text { lifetime }(\mathrm{s})\end{array}$ & $\begin{array}{c}P_{\text {tot }} \\
\left(\mathrm{erg} \mathrm{cm}^{-2} \mathrm{~s}^{-1}\right)\end{array}$ & $\begin{array}{c}P_{\text {tot }} \text { using } \\
E_{\text {Min }}=10^{21}\end{array}$ \\
\hline Set 1 & $2013 / 08 / 31$ & $7.86 \times 10^{22}$ & $3.27 \times 10^{25}$ & $1.93 \pm 0.04$ & $\sim 36$ & $1.72 \times 10^{6}$ & $2.72 \times 10^{6}$ \\
Set 2 & $2013 / 12 / 17$ & $9.33 \times 10^{22}$ & $6.46 \times 10^{26}$ & $1.96 \pm 0.02$ & $\sim 60$ & $2.24 \times 10^{6}$ & $3.66 \times 10^{6}$ \\
Set 3 & $2015 / 04 / 16$ & $1.41 \times 10^{23}$ & $2.58 \times 10^{26}$ & $2.03 \pm 0.02$ & $\sim 60$ & $2.47 \times 10^{6}$ & $4.67 \times 10^{6}$ \\
Set 4 & $2015 / 11 / 12$ & $2.02 \times 10^{23}$ & $1.75 \times 10^{25}$ & $2.33 \pm 0.04$ & $\sim 39$ & $1.06 \times 10^{6}$ & $5.40 \times 10^{6}$ \\
Set 5 & $2015 / 11 / 12$ & $3.38 \times 10^{23}$ & $1.22 \times 10^{26}$ & $2.20 \pm 0.05$ & $\sim 60$ & $6.74 \times 10^{5}$ & $2.35 \times 10^{6}$ \\
Set 6 & $2017 / 03 / 26$ & $7.05 \times 10^{22}$ & $1.64 \times 10^{24}$ & $2.29 \pm 0.07$ & $\sim 15$ & $2.13 \times 10^{5}$ & $7.53 \times 10^{5}$ \\
Set 7 & $2017 / 03 / 26$ & $7.41 \times 10^{22}$ & $1.04 \times 10^{25}$ & $2.25 \pm 0.05$ & $\sim 9$ & $3.45 \times 10^{5}$ & $9.46 \times 10^{5}$ \\
Set 8 & $2017 / 05 / 01$ & $1.47 \times 10^{23}$ & $7.17 \times 10^{25}$ & $2.08 \pm 0.01$ & $\sim 36$ & $6.17 \times 10^{5}$ & $1.10 \times 10^{6}$ \\
Set 9 & $2016 / 06 / 06$ & $2.61 \times 10^{23}$ & $1.35 \times 10^{26}$ & $2.03 \pm 0.02$ & $\sim 36$ & $8.22 \times 10^{5}$ & $1.54 \times 10^{6}$ \\
Set 10 & $2014 / 01 / 07$ & $1.94 \times 10^{23}$ & $7.60 \times 10^{25}$ & $1.98 \pm 0.02$ & $\sim 60$ & $3.15 \times 10^{6}$ & $6.01 \times 10^{6}$ \\
Set 11 & $2016 / 01 / 14$ & $5.88 \times 10^{22}$ & $1.80 \times 10^{25}$ & $2.07 \pm 0.03$ & $\sim 33$ & $1.28 \times 10^{6}$ & $2.77 \times 10^{6}$ \\
Set 12 & $2016 / 01 / 19$ & $7.76 \times 10^{22}$ & $4.35 \times 10^{24}$ & $2.25 \pm 0.07$ & $\sim 33$ & $9.70 \times 10^{5}$ & $4.54 \times 10^{6}$ \\
\hline
\end{tabular}

per unit area can be written as follows:

$P_{1400}=\int_{E_{\min }}^{E_{\max }} f(E) E d E=\left.\frac{f_{0}}{-\gamma+2} E^{-\gamma+2}\right|_{E_{\min }} ^{E_{\max }}$,

where $f_{0}$ is the normalization factor that can be calculated from the y-intercept of the energy distribution. $E_{\min }$ and $E_{\max }$ represent the observed minimum and maximum energy of the impulsive events from any given data set.

In order to calculate the total energy of the bursts, we must first estimate the Si IV $1394 \AA$ emission. This can be done from the SJI $1400 \AA$ channel by multiplying the burst energy obtained using our algorithm by 0.6 (i.e. assuming that around of the $\sim 60$ per cent of the SJI $1400 \AA$ intensity is contributed by Si IV $1394 \AA$ emission; Hansteen et al. 2014). Once the Si IV $1394 \AA$ emission had been estimated, we calculated the total radiative power $\left(P_{\text {tot }}\right)$ per unit area using the equation:

$\log \left(P_{\text {tot }}\right)=2.69+1.08 \times \log \left(P_{\text {Si_IV }}\right)$.

This is based on work by Bruner \& McWhirter (1988) and Doyle (1996), who produced emission measure distributions for different solar regions (ARs, coronal holes, sunspots, 'quiescent' regions, and flares) plus a range of late-type stars based on data from the Hubble Space Telescope's International Ultraviolet Explorer and found a linear correlation between the total radiative output and that from a single spectral line. The typical error is \pm 40 percent (Bruner \& McWhirter 1988). The details of this calculation and the total radiative power obtained for all data sets are shown in Table 3 . The derived values are typically only $10-25$ per cent of what is required in thermal energy to sustain the corona. If we were to extend the power-law distributions down to an energy of $10^{21} \mathrm{erg}$ (as given in the final column in Table 3), and assume parity between the radiative energies reported here and thermal energy released by the impulsive events, then we would have approximately $25-50$ per cent of the required energy input to account for coronal heating.

Finally, we attempt to provide a more general answer as to the power-law index of these impulsive bursts in solar ARs. In order to do this, we combined all of the data sets studied here in two ways. The first method combined the results obtained from each data set when applying a minimum event lifetime of $60 \mathrm{~s}$. The second method considered only data sets that facilitated a minimum lifetime condition of less than approximately $35 \mathrm{~s}$. The intensity threshold was set to $3 \sigma$ and the area threshold was set to $>3$ pixels for both of these methods. In the top panel of Fig. 6, the derived distribution from the first method is plotted. The noise within the data is extremely low; however, the power-law index is well below 2 . When only data sets that could allow us to probe events with sub-minute lifetimes were studied, the power-law index increased such that $\gamma>2$. This result indicates that the analysis of extremely high-cadence IRIS SJI 1400 $\AA$ data in the future may provide further insights into the energy input into the outer atmosphere from small-scale impulsive bursts.

\section{DISCUSSION AND SUMMARY}

In this article, we have studied the effects of minimum area conditions, temporal resolution, intensity thresholding, and structuring within a FOV on the returned power-law indices of distributions of impulsive brightening events in the transition regions of solar ARs. We have studied 12 IRIS SJI data sets (detailed in Table 1) using the automated detection algorithm described in Section 2. Our results indicate that the minimum area condition has a limited influence on the power-law index, with the main effect of decreasing the minimum area being that one is able to observe lower energy events along the same power law (see Figs 3 and 5). The structuring within the FOV has a large effect on the $\gamma$ value with regions dominated by sunspots having smaller power-law indices than regions dominated by plage. It is interesting to note that if we select an intensity threshold of $3 \sigma$ above the background for impulsive event detections, then three out of eight plage data sets have power-law indices higher than 2 for both 60-s and 110-s lifetime thresholds (see Table 2).

In order to better understand the effects of cadence on the powerlaw indices, we also applied the algorithm to all data sets with a minimum lifetime value of three time-steps. With this criteria, nine of the 12 data sets returned $\gamma>2$ indicating that cadence is an important factor for power-law determination (see Table 3). The effects of cadence on the returned power law are best shown in Fig. 6, where combining all data sets with a minimum lifetime of $60 \mathrm{~s}$ returns a lower $\gamma$ value than combining all data sets with a 35-s minimum lifetime. Using the power-law indices obtained with a minimum lifetime of three times the cadence, we calculated the total radiative energy of the observed impulsive events for each data set. The estimated total radiative power obtained for these data sets is typically only 25 per cent of the thermal energy required to maintain the temperature of the corona (see Table 3). Although this would not be sufficient to heat solar plage regions, it would constitute a major contribution. However, if the derived slopes extend to energies 

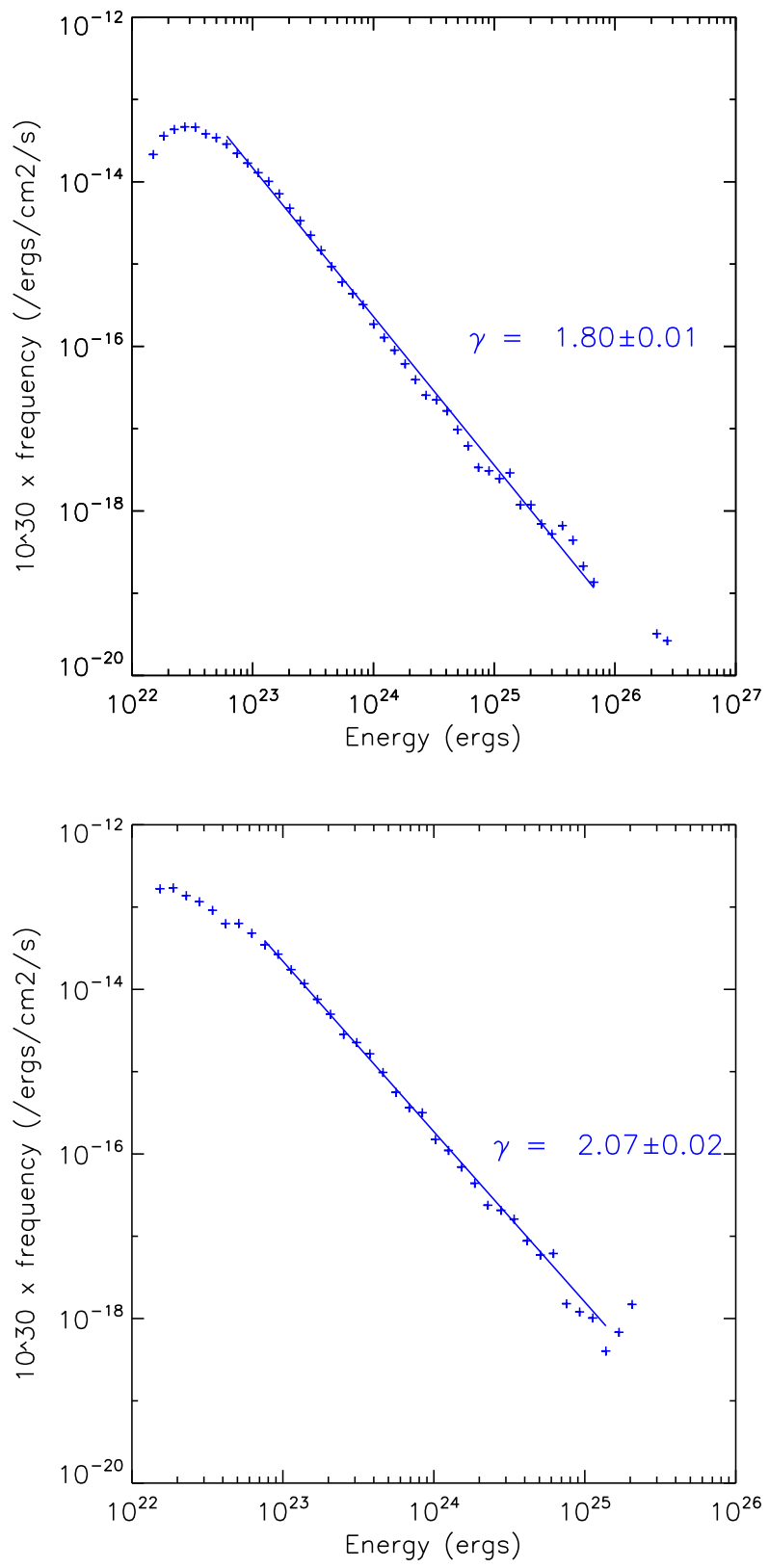

Figure 6. Combined energy distribution of all 12 data sets using a cadence of $\sim 60 \mathrm{~s}$ (top panel). Combined energy distribution from all data sets studied with a cadence of less than $\sim 35$ s, i.e. for Data sets $1,4,6,7,8,9,11$, and 12 (bottom panel).

of $10^{21} \mathrm{erg}$, then the radiative energy would be at parity with the thermal energy required for coronal heating.

In all of the regions studied, instrument sensitivity has a major impact on the detections at lower energies. Mondal et al. (2020) recently used the Murchison Widefield Array to observe the quiet Sun at radio frequencies. They detected impulsive emission with flux densities of about two orders of magnitude lower than the earlier attempts (a few mSFU where a Solar Flux Unit is $10^{-19} \mathrm{erg} \mathrm{s}^{-1}$ $\mathrm{cm}^{-2} \mathrm{~Hz}^{-1}$ ). These impulsive events have duration of $\sim 1 \mathrm{~s}$ and are present throughout the quiet solar corona. The estimate of the energy, which must be dumped in the corona to generate these impulsive events, is consistent with that required for coronal heating. In the events studied by these authors, the power-law indices were all $>2$.
Combining this with our analysis implies that high cadence is an essential requirement for future analyses. In the future, the highest cadence IRIS SJI data sets available should be analysed in order to better understand the presence of impulsive brightenings at the smallest energy scales.

The Spectral Imaging of the Coronal Environment (SPICE) instrument onboard Solar Orbiter will observe a range of ultraviolet lines, the strongest being O VI $1031.9 \AA$ with an estimated 8000 photons pixel ${ }^{-1} \mathrm{~s}^{-1}$ in an AR (Anderson et al. 2020). This would allow confirmation that brightening events from plage regions can account for the heating of the corona. Another upcoming facility is the Daniel K. Inouye Solar Telescope (DKIST), which will have a spatial resolution a factor of 3 better than currently available in the lower solar atmosphere. In the photosphere/lower chromosphere, Reid et al. (2016) analysed EBs observed in $\mathrm{H} \alpha$-line profiles recorded by the Swedish Solar Telescope (Scharmer et al. 2003) using automated methods. These observations also took place in ARs; therefore, it is possible to compare their statistics with the impulsive brightenings detected in the upper atmosphere of the AR. The energies of the EBs ranged from $10^{23} \mathrm{erg}$ to $10^{26} \mathrm{erg}$. The power-law index obtained for energies of the EBs was 1.67. Nelson et al. (2013) also looked at brightenings in the $\mathrm{H} \alpha$ line with the Dunn Solar Telescope but reduced the required intensity enhancement thereby detecting many more impulsive events. The radiative energy covered was from $2 \times 10^{22}$ to $4 \times 10^{25} \mathrm{erg}$ with a slope of 2.14. Both of these power-law indices are comparable to those found here for a range of data sets. With the improved spatial resolution offered by DKIST, we will be able to resolve very small-scale flaring events.

\section{ACKNOWLEDGEMENTS}

Armagh Observatory and Planetarium is core funded by the Northern Ireland Executive through the Department for Communities. IRIS is a NASA small explorer mission developed and operated by LMSAL with mission operations executed at NASA Ames Research Center and major contributions to downlink communications funded by ESA and the Norwegian Space Centre. We would like to thank the AIA and IRIS teams for providing valuable data. CJN and MM acknowledge support from STFC under grant no. ST/P000304/1. We would like to thank Dr. Aaron Reid for discussions on the topic of Ellerman Bombs.

\section{DATA AVAILABILITY}

The data sets were derived from sources in the public domain: [IRIS; https://iris.lmsal.com/, AIA; http://jsoc.stanford.edu/].

\section{REFERENCES}

Alfvén H., 1947, MNRAS, 107, 211

Anderson M. et al., 2020, A\&A, 642, A14

Antiochos S. K., Karpen J. T., DeLuca E. E., Golub L., Hamilton P., 2003, ApJ, 590, 547

Aschwanden M. J., Parnell C. E., 2002, ApJ, 572, 1048

Ayres T. R. et al., 1995, ApJS, 96, 223

Bowness R., Hood A. W., Parnell C. E., 2013, A\&A, 560, A89

Brueckner G. E., Bartoe J. D. F., 1983, ApJ, 272, 329

Bruner M. E., McWhirter R. W. P., 1988, ApJ, 326, 1002

Cargill P. J., Klimchuk J. A., 2004, ApJ, 605, 911

De Pontieu B. et al., 2014, Sol. Phys., 289, 2733

Doyle J. G., 1996, A\&A, 307, 162

Doyle J. G., Butler C. J., 1985, Nature, 313, 378

Ellerman F., 1917, ApJ, 46, 298 
Golub L. et al., 1999, Phys. Plasmas, 6, 2205

Graham D. R., De Pontieu B., Testa P., 2019, ApJ, 880, L12

Hansteen V. et al., 2014, Science, 346, 1255757

Huang Z., Madjarska M. S., Scullion E. M., Xia L. D., Doyle J. G., Ray T., 2017, MNRAS, 464, 1753

Huang Z. et al., 2018, ApJ, 854, 80

Hudson H. S., 1991, Sol. Phys., 133, 357

Jess D. B. et al., 2019, ApJ, 871, 133

Krucker S., Benz A. O., 1998, ApJ, 501, L213

Lemen J. R. et al., 2012, Sol. Phys., 275, 17

Levine R. H., 1974, ApJ, 190, 457

Mondal S., Oberoi D., Mohan A., 2020, ApJL, 895, L39

Nelson C. J., Doyle J. G., Erdélyi R., Huang Z., Madjarska M. S., Mathioudakis M., Mumford S. J., Reardon K., 2013, Sol. Phys., 283, 307

Nelson C. J., Scullion E. M., Doyle J. G., Freij N., Erdélyi R., 2015, ApJ, 798, 19

Nelson C. J., Doyle J. G., Erdélyi R., 2016, MNRAS, 463, 2190

Nelson C. J., Freij N., Reid A., Oliver R., Mathioudakis M., Erdélyi R., 2017, ApJ, 845, 16

Parker E. N., 1988, ApJ, 330, 474

Parnell C. E., Jupp P. E., 2000, ApJ, 529, 554
Parnell C. E., Maclean R. C., Haynes A. L., 2010, ApJ, 725, L214

Peter H. et al., 2014, Science, 346, 1255726

Rappazzo A. F., Velli M., Einaudi G., Dahlburg R. B., 2008, ApJ, 677, 1348

Rathore B., Carlsson M., 2015, ApJ, 811, 80

Rathore B., Carlsson M., Leenaarts J., De Pontieu B., 2015, ApJ, 811, 81

Reid A., Mathioudakis M., Doyle J. G., Scullion E., Nelson C. J., Henriques V., Ray T., 2016, ApJ, 823, 110

Rouppe van der Voort L. H. M., Rutten R. J., Vissers G. J. M., 2016, A\&A, 592, A100

Scharmer G. B., Bjelksjo K., Korhonen T. K., Lindberg B., Petterson B., 2003, Proc. SPIE Conf. Ser. Vol. 4853, The 1-Meter Swedish Solar Telescope. SPIE, Bellingham, p. 341

Shimizu T., 1995, PASJ, 47, 251

Testa P. et al., 2013, ApJ, 770, L1

Vissers G. J. M., Rouppe van der Voort L. H. M., Rutten R. J., 2013, ApJ, 774,32

Withbroe G. L., Noyes R. W., 1977, ARA\&A, 15, 363

Young P. R. et al., 2018, Space Sci. Rev., 214, 120

This paper has been typeset from a $\mathrm{T}_{\mathrm{E}} \mathrm{X} / \mathrm{L} \mathrm{T}_{\mathrm{E}} \mathrm{X}$ file prepared by the author. 